\title{
Search for supersymmetry in events involving third generation squarks and sleptons with ATLAS
}

\author{
Katarina Pajchel \\ on behalf of the ATLAS Collaboration
}

University of Oslo

CIPANP, May 29 - June 3, 2012

St. Petersburg, Florida
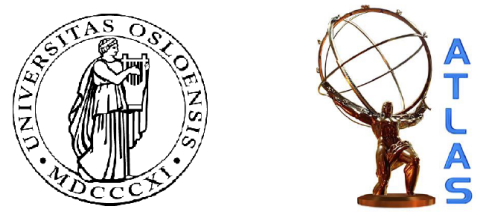


\section{Introduction}

- Supersymmetry (SUSY) - focus on R-parity conserving models $\triangleright$ Stable LSP - large missing transverse momentum $E_{T}^{\text {miss }}$

- Left- and right-handed gauge states mix to form mass eigenstates

$\triangleright$ Large mixing $\rightarrow$ lighter 3rd generation squarks and sleptons

$\triangleright$ Sub-TeV stop/sbottom favored by the naturalness argument

- Scalar top $\left(\tilde{t}_{1}\right)$ and bottom $\left(\tilde{b}_{1}\right)$ - direct pair production or in $\tilde{g}$ - pair production

- Scalar tau $\left(\tilde{\tau}_{1}\right)$ - lightest slepton, produced in cascade decays
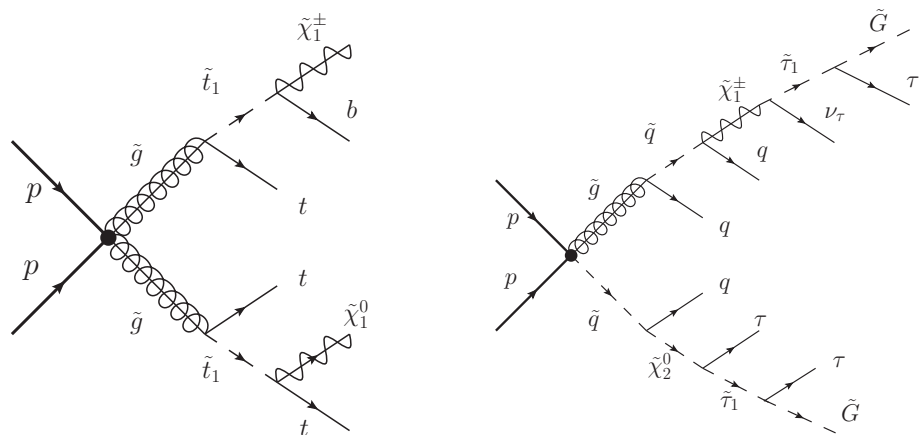


\section{General approach in ATLAS SUSY searches}

- Trigger selection - three levels with increasing refinement

- Event quality pre-selection - Integrated luminosity $2.05 \mathrm{fb}^{-1}$

- Offline object selection assures plateau trigger efficiency

- Signal regions (SR) optimized for characteristic final states using bench mark models. Defined by: jets, b-jets, leptons, $E_{T}^{\text {miss }}$,

$m_{\text {eff }}=E_{T}^{\text {miss }}+\sum_{\text {jets }} p_{T}\left(+\sum_{\text {lep }} p_{T}\right) \quad m_{T}=\sqrt{2 p_{T}^{l} E_{T}^{\text {miss }}-2 \mathbf{p}_{T}^{\prime} \cdot \mathbf{p}_{T}^{\text {miss }}}$

- Background estimation

$\triangleright$ Data- and semi-data driven methods (details in backup)

- Systematic uncertainties

$\triangleright$ Detector related and theoretical

- In absence of significant excess: set model independent 95\% C.L. upper limits on contribution from new physics using the $C L_{S}$ prescription

- Results interpreted within context of various models

$\triangleright 95 \%$ C.L. exclusion limits on model parameters, particle masses 


\section{Searches for SUSY with jets, missing transverse momentum and one - two tau leptons}

\section{Publications:}

- $1 \tau+E_{T}^{\text {miss }}+$ jets with $2 \mathrm{fb}^{-1}$ : arXiv: 1204.3852 submitted to PLB

- $2 \tau+E_{T}^{\text {miss }}+$ jets with $2 \mathrm{fb}^{-1}$ : arXiv: 1203.6580 submitted to PLB 


\section{Final states with $\geq 1$ or $\geq 2 \tau$ jets and $E_{T}^{\text {miss }}$}

- Final states with taus studied in context of GMSB - light $\tilde{\tau}_{1}$

- Two analyses - common event preselection

$\triangleright E_{T}^{\text {miss }}$ and $p_{T}\left(j_{1}\right)>130 \mathrm{GeV}$ (trigger offline)

$\triangleright$ Lepton $(e, \mu)$ veto

- Backgrounds: top, $W+$ jets, $Z+$ jets, multijet, real and fake $\tau$

- $\geq 1$ tight hadronic $\tau, p_{T}>20 \mathrm{GeV}$

- $m_{T}^{\tau}>110 \mathrm{GeV}$

- $m_{\text {eff }}>600 \mathrm{GeV}$

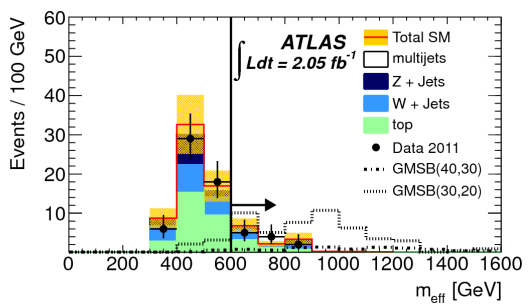

- $\geq 2$ loose hadronic $\tau, p_{T}>20 \mathrm{GeV}$

- $m_{\text {eff }}>700 \mathrm{GeV}$

- $m_{T}^{\tau_{1}}+m_{T}^{\tau_{2}}>80 \mathrm{GeV}$

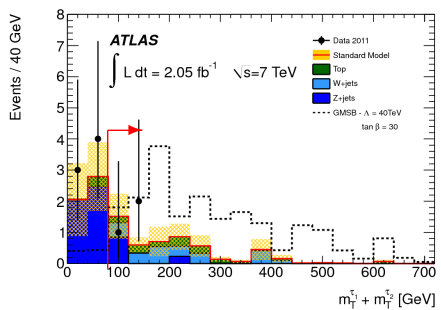




\section{Final states with taus: Interpretation}

- Data in agreement with SM prediction

$\triangleright$ Upper limit at 95\% C.L.

- GMSB - grid

$\triangleright$ LSP is $\tilde{G}, m_{\tilde{G}}<<1 \mathrm{keV}$

$\triangleright M_{\text {mess }}=250 \mathrm{TeV}, N_{5}=3, \mu>0$, $C_{\text {grav }}=1$

- Free model parameters:

$\triangleright \wedge$ - scale of the SUSY breaking

$\triangleright \tan \beta$ - ratio of the two Higgsino doublet vacuum expectation value

- Gluino and squark production, decay to NLSP $-\tilde{\tau}_{1}$ in most of the parameter space $\rightarrow$ LSP + SM particles

- Exclusion limits on GMSB parameters

$$
\begin{aligned}
\triangleright & 1 \tau: \Lambda<30 \mathrm{TeV}, \\
& \text { best } \Lambda=43 \mathrm{TeV} \text { for high } \tan \beta \\
\triangleright & 2 \tau: \Lambda<32 \mathrm{TeV}, \\
& \text { best } \Lambda=47 \mathrm{TeV} \tan \beta=37
\end{aligned}
$$

ATLAS dilepton $(e, \mu)$ OS with $1 \mathrm{fb}^{-1}$ (ATLAS-CONF-2011-156)
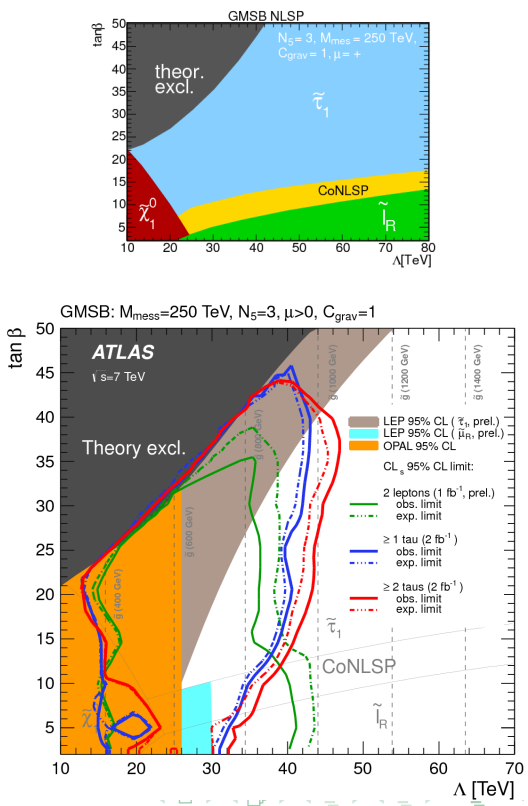

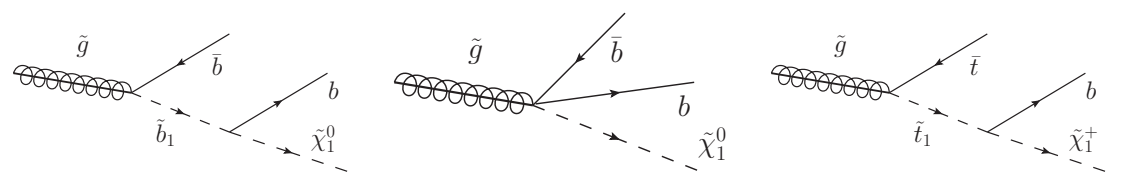

\section{Searches for SUSY with b-jets, missing transverse momentum and 0 or 1 leptons}

Publications:

- arXiv:1203.6193 Accepted by PRD

- Gluino mediated sbottom production $\tilde{g} \rightarrow \tilde{b}_{1} b \rightarrow b \bar{b} \tilde{\chi}_{1}^{0}, \tilde{g} \rightarrow b \bar{b} \tilde{\chi}_{1}^{0}$ via $\tilde{b}_{1}^{*}$

- Gluino mediated stop production $\tilde{g} \rightarrow \tilde{t}_{1} t \rightarrow t \bar{t} \tilde{\chi}_{1}^{0}, \tilde{g} \rightarrow t \bar{t} \tilde{\chi}_{1}^{0}$ via $\tilde{t}_{1}^{*}$

- A search sensitive to many scenarios 


\section{Final states with b-jets, jets, $E_{T}^{\text {miss }}, 0-1$ leptons}

- 0-lepton channel optimized for $\tilde{g} \rightarrow b \bar{b} \tilde{\chi}_{1}^{0}, 1$-lepton for $\tilde{g} \rightarrow t \bar{t} \tilde{\chi}_{1}^{0}$,

\begin{tabular}{l|c|c}
\hline \hline Pre-selection & Signal Region name & Selection \\
\hline \hline & SR0-A1 & at least one $b$-tag, $m_{\text {eff }}>500 \mathrm{GeV}$ \\
no leptons, at least three jets, & SR0-B1 & at least one $b$-tag, $m_{\text {eff }}>700 \mathrm{GeV}$ \\
$p_{\mathrm{T}}(j 1)>130 \mathrm{GeV}, p_{\mathrm{T}}(j 2, j 3)>50 \mathrm{GeV}$, & SR0-C1 & at least one $b$-tag, $m_{\text {eff }}>900 \mathrm{GeV}$ \\
$E_{\mathrm{T}}^{\text {miss }}>130 \mathrm{GeV}, E_{\mathrm{T}}^{\text {miss }} / m_{\text {eff }}>0.25$, & SR0-A2 & at least two $b$-tags, $m_{\text {eff }}>500 \mathrm{GeV}$ \\
$\Delta \phi_{\min }>0.4$ & SR0-B2 & at least two $b$-tags, $m_{\text {eff }}>700 \mathrm{GeV}$ \\
& SR0-C2 & at least two $b$-tags, $m_{\text {eff }}>900 \mathrm{GeV}$ \\
\hline one lepton, at least four jets & SR1-D & $m_{\text {eff }}>700 \mathrm{GeV}$ \\
$p_{\mathrm{T}}(j 1)>60 \mathrm{GeV}, p_{\mathrm{T}}(j 2, j 3, j 4)>50 \mathrm{GeV}$, & SR1-E & $m_{\text {eff }}>700 \mathrm{GeV}, E_{\mathrm{T}}^{\text {miss }}>200 \mathrm{GeV}$ \\
$E_{\mathrm{T}}^{\text {miss }}>80 \mathrm{GeV}, m_{\mathrm{T}}>100 \mathrm{GeV}$, & & \\
at least one $b$-tag & & \\
\hline \hline
\end{tabular}

- SM background: top (single and in pair), $W / Z$ with heavy-flavor jets, multiple-jets
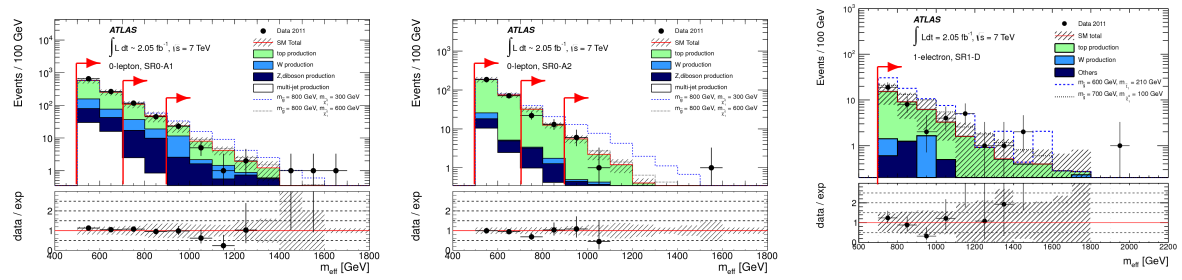


\section{Final states with b-jets, jets, $E_{T}^{\text {miss }}, 0-1$ leptons: Results}

0-lepton channel

\begin{tabular}{c|c|c|c||c||c}
\hline \hline SR & Top & W/Z & $\begin{array}{c}\text { QCD/ } \\
\text { di-boson }\end{array}$ & Total & Data \\
\hline SR0-A1 & $\begin{array}{c}705 \pm 110 \\
(725)\end{array}$ & $248 \pm 150$ & $53 \pm 21$ & $1000 \pm 180$ & 1112 \\
SR0-B1 & $\begin{array}{c}119 \pm 26 \\
(122)\end{array}$ & $67 \pm 42$ & $7.3 \pm 4.7$ & $190 \pm 50$ & 197 \\
SR0-C1 & $\begin{array}{c}22 \pm 9 \\
(22)\end{array}$ & $16 \pm 11$ & $1.5 \pm 1$ & $39 \pm 14$ & 34 \\
SR0-A2 & $\begin{array}{c}272 \pm 70 \\
(212)\end{array}$ & $22.5 \pm 15$ & $21 \pm 12$ & $316 \pm 72$ & 299 \\
SR0-B2 & $\begin{array}{c}47 \pm 11 \\
(37)\end{array}$ & $4.5 \pm 3$ & $2.8 \pm 1.7$ & $54 \pm 11$ & 43 \\
SR0-C2 & $\begin{array}{c}8.5 \pm 3 \\
(6.6)\end{array}$ & $0.8 \pm 1$ & $0.5 \pm 0.4$ & $9.8 \pm 3.2$ & 8 \\
\hline \hline
\end{tabular}

- Interpretation:

- MSSM Gluino-sbottom models: $m_{\tilde{g}}>m_{\tilde{b}_{1}}$

$\triangleright \tilde{g} \rightarrow \tilde{b}_{1} b,, \tilde{b}_{1} \rightarrow b \tilde{\chi}_{1}^{0}$

- Gbb: simplified scenario, $m_{\tilde{g}}<m_{\tilde{b}_{1}}$

$\triangleright \tilde{g}$ decay via $\tilde{b}_{1}^{*}, \tilde{b}_{1}^{*} \rightarrow b \tilde{\chi}_{1}^{0}$ 1-lepton channel

\begin{tabular}{c|c||c}
\hline \hline SR & SM background & Data \\
\hline SR1-D $(e)$ & $39 \pm 12(39)$ & 43 \\
SR1-D $(\mu)$ & $38 \pm 14(37)$ & 38 \\
SR1-E $(e)$ & $8.1 \pm 3.4(7.9)$ & 11 \\
SR1-E $(\mu)$ & $6.3 \pm 4.2(6.1)$ & 6 \\
\hline \hline
\end{tabular}

$\mathrm{MC}$ yields in parenthesis
- Interpretation:

- MSSM Gluino-stop models $m_{\tilde{g}}>m_{\tilde{t}_{1}}+m_{t}$ $\triangleright \tilde{g} \rightarrow \tilde{t}_{1} t, \tilde{t}_{1} \rightarrow b \tilde{\chi}_{1}^{ \pm}$

- Gtt: simplified scenario $m_{\tilde{g}}<m_{\tilde{t}_{1}}$

$\triangleright \tilde{g}$ decay via $\tilde{t}_{1}^{*}, \tilde{t}_{1}^{*} \rightarrow t \tilde{\chi}_{1}^{0}$ 


\section{Final states with b-jets, jets, $E_{T}^{m i s s}, 0-1$ leptons: Limits}
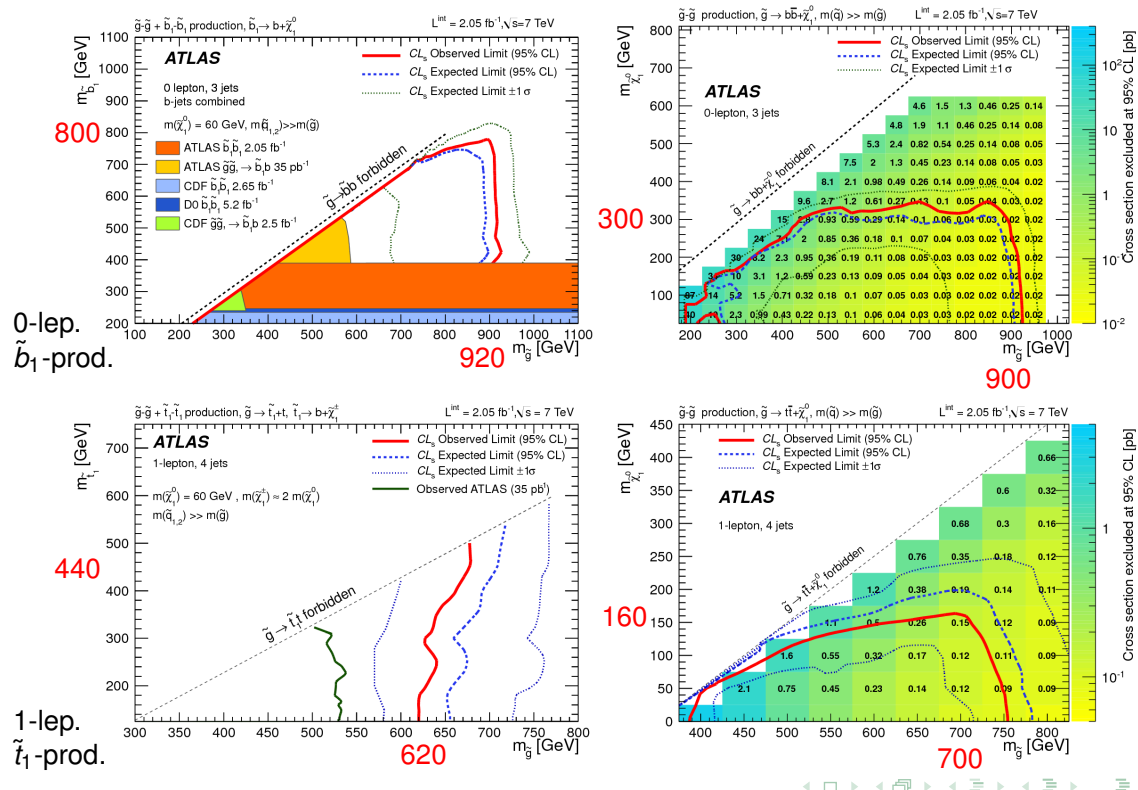


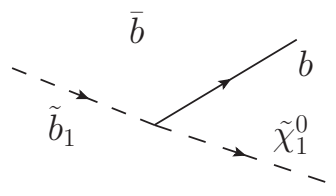

Searches for scalar bottom quark production $2 \mathrm{~b}$-jets, 0 leptons and $E_{T}^{\text {miss }}$

Publications:

- PRL 108 (2012) 181802

- Direct sbottom pair production, $\tilde{b}_{1} \rightarrow b \tilde{\chi}_{1}^{0}$ 


\section{Final states with $2 \mathrm{~b}$-jets and $E_{T}^{\text {miss }}$}

- Event selection:

- $p_{T}(j 1), E_{T}^{\text {miss }}>130 \mathrm{GeV}$ (trig.)

- Exactly 2 b-jets, lepton veto $(e, \mu)$

- Dominant SM backgrounds

- Signal region selection based on boost-corrected contransverse mass $m_{C T}^{2}\left(b_{1}, b_{2}\right)=\left[E_{T}\left(b_{1}\right)+E_{T}\left(b_{2}\right)\right]^{2}-\left[\mathbf{p}_{T}\left(b_{1}\right)-\mathbf{p}_{T}\left(b_{2}\right)\right]^{2}$

$\triangleright m_{C T}>100 \mathrm{GeV}$ : Top, $Z / W+$ heavy flavor

$\triangleright m_{C T}>150,200 \mathrm{GeV}$ : $Z / W+$ heavy flavor

- Sbottom pair production $\tilde{b}_{1} \rightarrow b \tilde{\chi}_{1}^{0}$ $m_{C T}$ endpoint at

$$
\frac{m\left(\tilde{b}_{1}\right)^{2}-m\left(\tilde{\chi}_{1}^{0}\right)^{2}}{m\left(\tilde{b}_{1}\right)}
$$

- $t \bar{t}$ production endpoint at $135 \mathrm{GeV}$

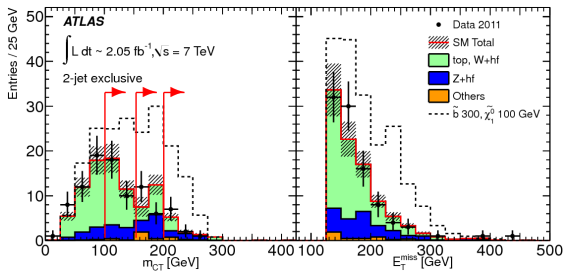

- 3 signal regions:

$m_{C T}>100,150,200 \mathrm{GeV}$ 


\section{Final states with 2 b-jets and $E_{T}^{m i s s: ~ R e s u l t s ~}$}

- Good agreement data and SM prediction

- Interpretation within model with $\tilde{b}$-pair production, assuming $\operatorname{BR}\left(\tilde{b}_{1} \rightarrow b \tilde{\chi}_{1}^{0}\right)=1$

- Exclusion limits in $\left(m_{\tilde{b}_{1}}-m_{\tilde{\chi}_{1}^{0}}\right)$ plane

$\triangleright m_{\tilde{b}_{1}}<390 \mathrm{GeV}$ for $m_{\tilde{\chi}_{1}^{0}}=0 \mathrm{GeV}$

$\triangleright m_{\tilde{\chi}_{1}^{0}}<120 \mathrm{GeV}$ for $275<m_{\tilde{b}_{1}}<350 \mathrm{GeV}$

\begin{tabular}{l|ccc|c|c}
\hline \hline$m_{\mathrm{CT}}$ & top, $W+\mathrm{hf}$ & $\begin{array}{c}Z+\mathrm{hf} \\
\mathrm{TF}\end{array}$ & $\begin{array}{c}\text { Others } \\
\mathrm{GCV}\end{array}$ & Total SM & Data \\
& $\begin{array}{c}\mathrm{TF} \\
(\mathrm{MC})\end{array}$ & $(\mathrm{MC})$ & & & \\
\hline 0 & $67 \pm 10$ & $23 \pm 8$ & $3.6 \pm 1.5$ & $94 \pm 16$ & 96 \\
& $(60 \pm 25)$ & $(16 \pm 9)$ & & $(80 \pm 35)$ & \\
100 & $36 \pm 10$ & $23 \pm 9$ & $3.1 \pm 1.6$ & $62 \pm 13$ & 56 \\
& $(34 \pm 16)$ & $(12 \pm 7)$ & & $(49 \pm 25)$ & \\
150 & $12 \pm 5$ & $12 \pm 6$ & $2.7 \pm 0.9$ & $27 \pm 8$ & 28 \\
& $(13 \pm 8)$ & $(8.3 \pm 4.7)$ & & $(24 \pm 13)$ & \\
200 & $3.2 \pm 1.6$ & $3.9 \pm 3.2$ & $1.0 \pm 0.9$ & $8.1 \pm 3.5$ & 10 \\
& $(4.1 \pm 3.4)$ & $(2.8 \pm 1.5)$ & & $(8.0 \pm 4.9)$ & \\
\hline \hline
\end{tabular}

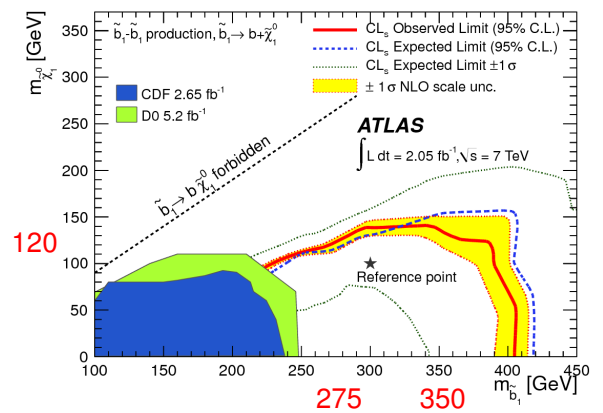




\section{Searches for scalar top quark production 2 leptons and $E_{T}^{\text {miss }}$}

Publications:

- 2 I SS + jets + $E_{T}^{\text {miss: }}$ arXiv:1203.5763 Submitted to PRL

- 2 I SFOS + jets $+E_{T}^{\text {miss: }}$ arXiv:1204.6736 Submitted to PLB 


\section{2 / SS + jets $+E_{T}^{\text {miss: }}$ gluino mediated stop production}

- Gluino is a Majorana-particle - equal probability for SS as for OS

- 2 leading leptons $(e, \mu) \mathrm{SS}, p_{T}>20 \mathrm{GeV}$

- $N_{\text {jet }} \geq 4, p_{T}(j 4)>50 \mathrm{GeV}$

$\triangleright$ SR1: $E_{T}^{\text {miss }}>150 \mathrm{GeV}$

$\triangleright$ SR2: $E_{T}^{\text {miss }}>150 \mathrm{GeV}$ and $m_{T}>100 \mathrm{GeV}$

- Results in SR2 interpreted within $\tilde{g} \tilde{g}$ production

$\triangleright$ Gtt simplified scenario: $m_{\tilde{g}}<m_{\tilde{t}_{1}}, \tilde{g} \rightarrow t \bar{t} \tilde{\chi}_{1}^{0}$ via $\tilde{t}_{1}^{*}$

$\triangleright$ MSSM scenario $\tilde{g} \rightarrow \tilde{t}_{1} t$ and $\tilde{t}_{1} \rightarrow b \tilde{\chi}_{1}^{ \pm}$

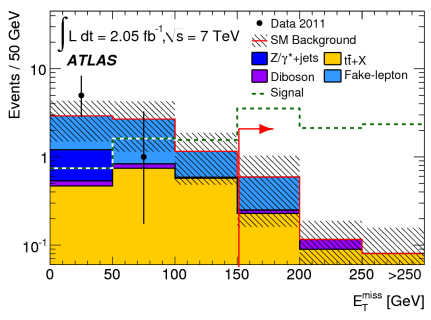

- No events observed with $E_{T}^{\text {miss }}>150 \mathrm{GeV}$ 


\section{I SS + jets $+E_{T}^{\text {miss: }}$ gluino mediated stop production}

- Excluded:

$$
m_{\tilde{g}}<650(720) \mathrm{GeV} \text { for } m_{\tilde{\chi}_{1}^{0}}<215(100) \mathrm{GeV} \quad m_{\tilde{g}}<670 \mathrm{GeV} \text { for } m_{\tilde{t}_{1}}<460 \mathrm{GeV}
$$
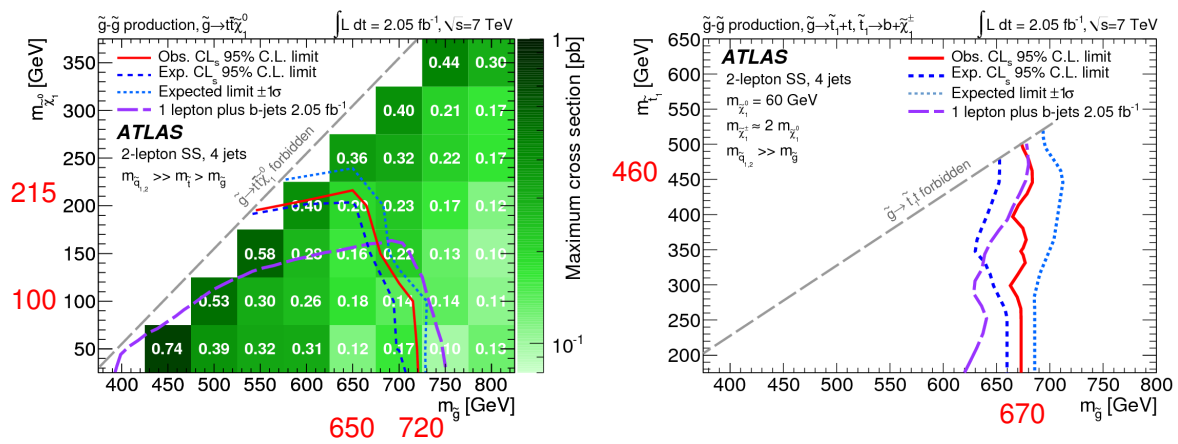


\section{I SFOS + jets $+E_{T}^{\text {miss: }}$ direct stop, GMSB}

- Direct stop pair production assuming GMSB: $\tilde{\chi}_{1}^{0}$ higgsino-like NLSP

$\triangleright$ Decay: $\quad \tilde{t}_{1} \rightarrow b \tilde{\chi}_{1}^{ \pm}$or $\tilde{t}_{1} \rightarrow t \tilde{\chi}_{1,(2)}^{0} \quad \tilde{\chi}_{1}^{0} \rightarrow Z \tilde{G}$ branching ratio 1-0.65

- SFOS pair with $86<m_{\ell^{+} \ell^{-}}<96 \mathrm{GeV}$

- At least: $p_{T}(j 2)>50 \mathrm{GeV}$ one b-tagged

$\triangleright \mathrm{SR} 1 E_{T}^{\text {miss }}>50 \mathrm{GeV} \mathrm{SR} 2 E_{T}^{\text {miss }}>80 \mathrm{GeV}$

- Background: Top, $Z+$ heavy-flavor jets

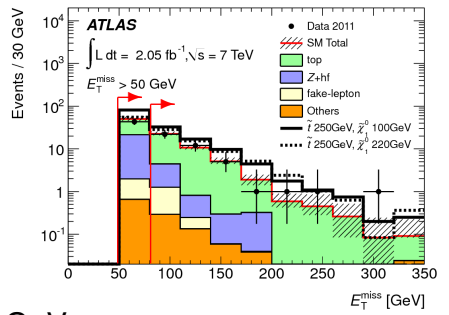

- Excluded: $m_{\tilde{t}_{1}}<310 \mathrm{GeV}$ for $115<m_{\tilde{\chi}_{1}^{0}}<230 \mathrm{GeV}$

\begin{tabular}{ccc}
\hline \hline \multicolumn{3}{c}{$e e+\mu \mu$} \\
\hline Data & 86 & 43 \\
SM & $92 \pm 19$ & $40.7 \pm 6.0$ \\
\hline top & $64.3 \pm 7.7$ & $34.8 \pm 5.0$ \\
$Z+$ hf & $24 \pm 16$ & $4.2 \pm 3.2$ \\
fake lepton & $2.4 \pm 0.9$ & $1.1 \pm 0.6$ \\
Others & $1.2 \pm 1.2$ & $0.6 \pm 0.6$ \\
\hline \multicolumn{3}{c}{} \\
95\% C.L. upper limits: observed (expected) \\
\hline events & $37.2(40.6)$ & $19.8(17.8)$ \\
visible $\sigma$ [fb] & $18.2(19.8)$ & $9.7(8.7)$ \\
\hline
\end{tabular}

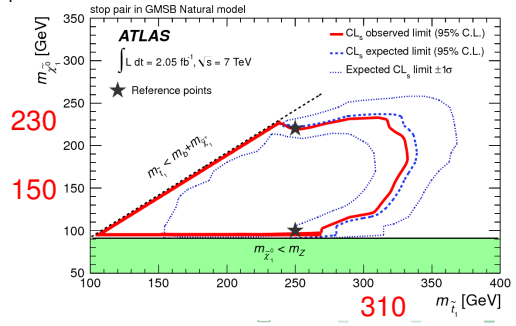




\section{Hunt for new phenomena using large jet multiplicity and $E_{T}^{\text {miss }}$}

Publications:

- 6-9 jets + $E_{T}^{\text {miss: }}$ : ATLAS-CONF-2012-037

- Full 2011 data set $4.7 \mathrm{fb}^{-1}$ 


\section{Multi-jets: Event selection}

- Gluino pair production in scenarios with $m_{\tilde{g}}<m_{\tilde{q}}$, possibly dominant decay:

$$
\tilde{g}+\tilde{g} \rightarrow\left(t+\tilde{t}+\tilde{\chi}_{1}^{0}\right)+\left(t+\tilde{t}+\tilde{\chi}_{1}^{0}\right)
$$

\begin{tabular}{|l||c|c|c|c|c|c|}
\hline Signal region & $\mathbf{7 j 5 5}$ & $\mathbf{8 j 5 5}$ & $\mathbf{9 j 5 5}$ & $\mathbf{6 j 8 0}$ & $\mathbf{7 j 8 0}$ & $\mathbf{8 j 8 0}$ \\
\hline \hline Isolated leptons $(e, \mu)$ & \multicolumn{7}{|c|}{$=0$} \\
\hline Jet $p_{T}$ & \multicolumn{7}{|c|}{$>55 \mathrm{GeV}$} & \multicolumn{5}{|c|}{$>80 \mathrm{GeV}$} \\
\hline Jet $|\eta|$ & \multicolumn{7}{|c|}{$<2.8$} \\
\hline Number of jets & $\geq 7$ & $\geq 8$ & $\geq 9$ & $\geq 6$ & $\geq 7$ & $\geq 8$ \\
\hline$E_{\mathrm{T}}^{\text {miss }} / \sqrt{H_{T}}$ & \multicolumn{7}{|c|}{$>4 \mathrm{GeV}^{1 / 2}$} \\
\hline
\end{tabular}

- $E_{T}^{\text {miss }} / \sqrt{H_{T}}$ - estimate of the $E_{T}^{\text {miss }}$ significance

- $H_{T}=\sum_{i} p_{T}\left(\right.$ jet $\left._{i}\right)$

- Background:

$\triangleright$ Multi-jet production, including fully hadronic top

$\triangleright$ Leptonic top, $Z / W+$ jets

- Multi-jet background

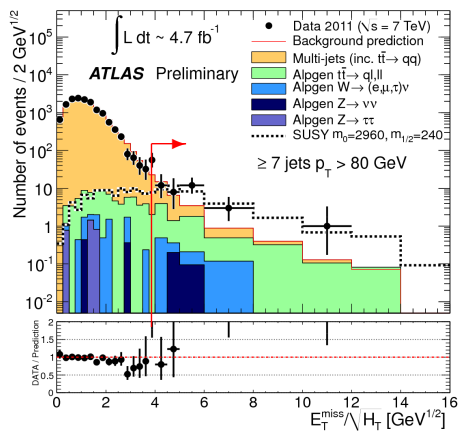

$\triangleright$ Fully data driven estimate 


\section{Multi-jets: Results}

\begin{tabular}{|c|c|c|c|c|c|c|}
\hline Signal regions & $7 \mathrm{j} 55$ & $8 \mathrm{j} 55$ & $9 \mathrm{j} 55$ & $6 \mathrm{j} 80$ & $7 \mathrm{j} 80$ & $8 \mathrm{j} 80$ \\
\hline Total SM & $167 \pm 34$ & $17 \pm 7$ & $1.9 \pm 0.8$ & $107 \pm 21$ & $8.6 \pm 2.5$ & $0.8 \pm 0.45$ \\
\hline Data & 154 & 22 & 3 & 106 & 15 & 1 \\
\hline
\end{tabular}

- Most discrepant 7j80 - p-value $=0.07$

- MSUGRA/CMSSM

$\tan \beta=10, A_{0}=0, \mu>0$

- Excluded: $m_{\text {gluino }}<850 \mathrm{GeV}$

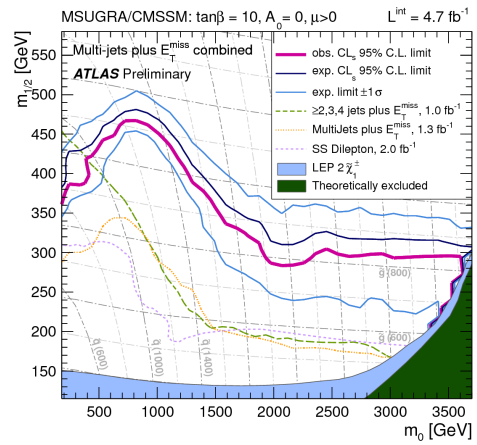

- Gtt: simplified scenario, $\tilde{g} \tilde{g}$ production

- $m_{\tilde{g}}<m_{\tilde{t}_{1}}$, decay $\tilde{g} \rightarrow t \bar{t} \tilde{\chi}_{1}^{0}$ via $\tilde{t}_{1}^{*}$

- $m_{\tilde{g}}<880 \mathrm{GeV}$ for $m_{\tilde{\chi}_{1}^{0}}<100 \mathrm{GeV}$

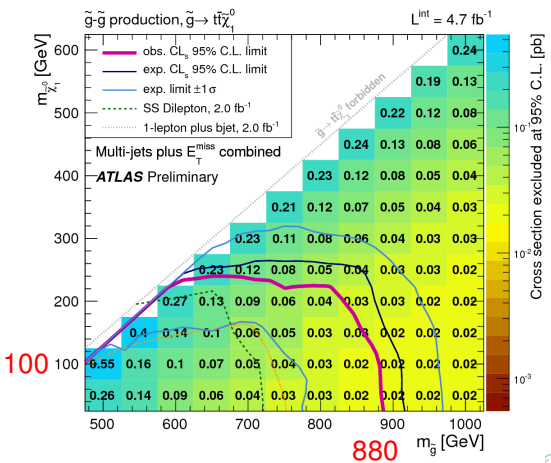




\section{Conclusions}

- ATLAS has a wide range of SUSY searches involving third generation squarks and sleptons

- No significant excess observed with respect to SM predictions

- 95\% C.L. upper limits on number of events and visible cross sections for BSM contributions are set

- Results are interpreted within a variety of SUSY models

\begin{tabular}{|c|c|c|c|}
\hline \multirow{5}{*}{ 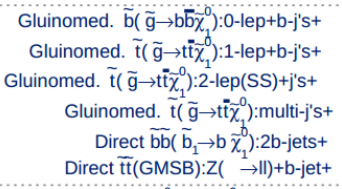 } & $E_{T \text {,miss }}$ & $L=2.1 \mathrm{fb} " \mathrm{~s}(2011)$ [ATLAS-CONF-2012-003] & 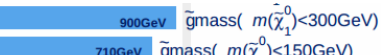 \\
\hline & $\begin{array}{l}E_{T \text {,miss }} \\
E_{T \text { miss }}\end{array}$ & $L=2.1 \mathrm{fb}^{-1}(2011)$ [ATLAS-CONF-2012-004] & $\begin{array}{l}710 \mathrm{GeV} \text { gmass }\left(m\left(\bar{\chi}_{1}\right)<150 \mathrm{GeV}\right) \\
650 \mathrm{Gev} \text { ğmass }\left(m\left(\tilde{\chi}_{1}^{0}\right)<210 \mathrm{GeV}\right)\end{array}$ \\
\hline & $E_{T \text { miss }}$ & $L=4.77 \mathrm{~b}^{-1}(2011)$ [ATLAS-CONF-2012-037] & $830 \mathrm{GeV}$ gmass $\left(m\left(\tilde{\chi}_{1}^{0}\right)<200 \mathrm{GeV}\right)$ \\
\hline & $E_{T, \text { miss }}$ & $L=2.16 b^{-1}(2011)[1112.3832]$ & $390 \mathrm{GeV}$ b̈mass $\left(m\left(\tilde{\chi}_{1}^{0}\right)<60 \mathrm{GeV}\right)$ \\
\hline & $E_{T, \text { miss. }}$ & $L=2.17 b^{21}(2011)$ [ATLAS-CONF-2012-036] & $310 \mathrm{GeV}$ tmass $\left(115<m\left(\tilde{\chi}_{1}^{0}\right)<230 \mathrm{GeV}\right)$ \\
\hline GMSB:1- $\tau+j^{\prime}{ }^{+}$ & $E_{T \text {,miss }}$ & $L=2.1 \mathrm{fb}^{-1}(2011)$ [ATLAS-CONF-2012-005] & $920 \mathrm{GeV}$ ḡmass $(\tan \beta>20)$ \\
\hline GMSB:2- $\tau+j$ 's+ & $E_{T \text {,miss }}$ & $L=2.1 \mathrm{fb}^{-1}(2011)$ [ATLAS-CONF-2012-002] & $990 \mathrm{Gev}$ g̃mass $(\tan \beta>20)$ \\
\hline
\end{tabular}

- Results with $4.7 \mathrm{fb}^{-1}$, the full 2011 data set, will be available soon

- Analysis of $8 \mathrm{TeV} 2012$ has data started 


\section{Back-up slides}




\section{Background estimation}

- Dominant background in most analyses: top and $Z / W+$ jets

- Semi-data driven method based on transfer factors $T_{f}^{b k g}$

$N_{S R}^{\text {bkg est }}=\frac{N_{S R}^{\text {bkg } M C}}{N_{C R}^{\text {kbg MC }}}\left(N_{C R}^{\text {data }}-N_{C R}^{\text {other } M C}\right)=T_{f}^{\text {bkg }}\left(N_{C R}^{\text {data }}-N_{C R}^{\text {other } M C}\right)$

- If event kinematics similar in SR and CR

$\triangleright$ Systematic uncertainties in numerator and denominator cancel out

- 0-lepton final states: Jet smearing method - data-driven

$\triangleright$ Contribution due to fake $E_{T}^{\text {miss }}$ - jet energy mismeasurement

$\triangleright$ Jet energies in events with small $E_{T}^{\text {miss }}$ (seed) smeared according to response functions

$\triangleright$ Large sample of pseudo-events passed through the analysis contribution estimated in a QCD-enhanced region

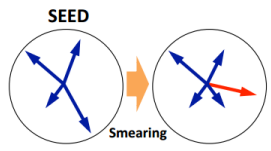

- 1/2-lepton final states: Matrix method - data-driven

$\triangleright$ Estimates contributions from non-prompt leptons arising from $b / c$-decay, jet, $\gamma$

$\triangleright$ Measure in data: probability of a "loose" lepton passing "tight" selection in "real" and "fake" enhanced control regions

$\triangleright$ Solve equation(s) which give the composition of SR in terms of "real" and "fake" leptons

- MC based predictions for sub-dominant modes 


\section{Final states with b-jets, jets, $E_{T}^{\text {miss }}, 0-1$ leptons: Results}

- Completing the results in slide 10,11

- Interpretation in Gtb: simplified scenario

$\triangleright \tilde{b}_{1}$ and $\tilde{t}_{1}$ lightest, $m_{\tilde{g}}<m_{\tilde{b}_{1}, \tilde{t}_{1}}$

$\triangleright \tilde{t}_{1} \rightarrow b \tilde{\chi}_{1}^{ \pm}, \tilde{b}_{1} \rightarrow t \tilde{\chi}_{1}^{ \pm}$

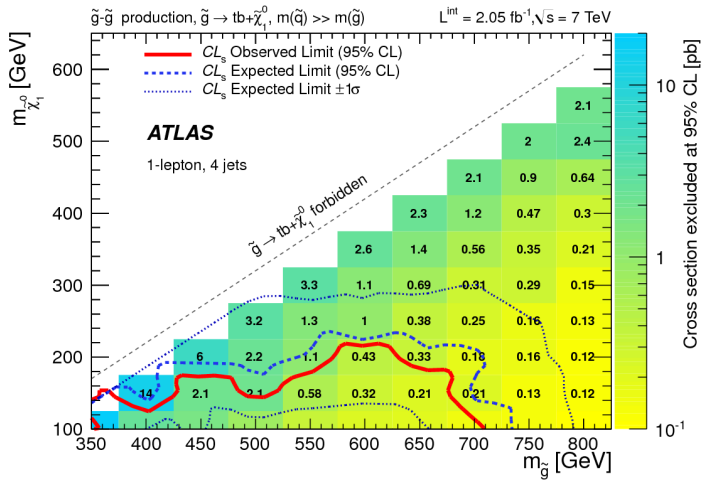




\section{Result table - Multi-jets}

\section{Hunt for new phenomena using large jet multiplicity and $E_{T}^{\text {miss }}$}

\begin{tabular}{|l||c|c|c|c|c|c|}
\hline Signal region & $\mathbf{7 j 5 5}$ & $\mathbf{8 j 5 5}$ & $\mathbf{9 j 5 5}$ & $\mathbf{6 j 8 0}$ & $\mathbf{7 j 8 0}$ & $\mathbf{8 j 8 0}$ \\
\hline \hline Multi-jets & $91 \pm 20$ & $10 \pm 3$ & $1.2 \pm 0.4$ & $67 \pm 12$ & $5.4 \pm 1.7$ & $0.42 \pm 0.16$ \\
\hline$t \bar{t} \rightarrow q \ell, \ell \ell$ & $55 \pm 18$ & $5.7 \pm 6.0$ & $0.70 \pm 0.72$ & $24 \pm 13$ & $2.8 \pm 1.8$ & $0.38 \pm 0.40$ \\
\hline$W+$ jets & $18 \pm 11$ & $0.81 \pm 0.72$ & $0+0.13$ & $13 \pm 10$ & $0.34 \pm 0.21$ & $0+0.06$ \\
\hline$Z+$ jets & $2.7 \pm 1.6$ & $0.05 \pm 0.19$ & $0+0.12$ & $2.7 \pm 2.9$ & $0.10 \pm 0.17$ & $0+0.13$ \\
\hline \hline Total Standard Model & $\mathbf{1 6 7} \pm \mathbf{3 4}$ & $\mathbf{1 7} \pm 7$ & $\mathbf{1 . 9} \pm \mathbf{0 . 8}$ & $\mathbf{1 0 7} \pm \mathbf{2 1}$ & $\mathbf{8 . 6} \pm \mathbf{2 . 5}$ & $\mathbf{0 . 8 0} \pm \mathbf{0 . 4 5}$ \\
\hline \hline Data & $\mathbf{1 5 4}$ & $\mathbf{2 2}$ & $\mathbf{3}$ & $\mathbf{1 0 6}$ & $\mathbf{1 5}$ & $\mathbf{1}$ \\
\hline \hline$N_{\mathrm{BSM}, \max }^{95 \%}(\exp )$ & 72 & 16 & 4.5 & 46 & 8.4 & 3.5 \\
$N_{\mathrm{BSM}, \max }^{95 \%}(\mathrm{obs})$ & 64 & 20 & 5.7 & 46 & 15 & 3.8 \\
\hline$\sigma_{\mathrm{BSM}, \max }^{95 \%} \cdot A \cdot \epsilon$ (exp) $[\mathrm{fb}]$ & 15 & 3.4 & 0.96 & 9.8 & 1.8 & 0.74 \\
$\sigma_{\mathrm{BSM}, \max }^{95 \%} \cdot A \cdot \epsilon$ (obs) $[\mathrm{fb}]$ & 14 & 4.2 & 1.2 & 9.8 & 3.2 & 0.81 \\
\hline$p_{\mathrm{SM}}$ & 0.64 & 0.27 & 0.28 & 0.52 & 0.07 & 0.43 \\
\hline
\end{tabular}

